CLNS-92/1162

CTP-2160

September 1992

\title{
Eluding the No-Hair Conjecture: Black Holes in Spontaneously Broken Gauge Theories
}

\author{
Brian R. Greene ${ }^{\dagger}$, Samir D. Mathur ${ }^{b}$ and Christopher M. O’Neill ${ }^{\dagger}$ \\ ${ }^{\dagger}$ Laboratory of Nuclear Studies \\ Cornell University \\ Ithaca, NY 14853 \\ ${ }^{b}$ Center for Theoretical Physics \\ Massachusetts Institute of Technology \\ Cambridge, MA 02139
}

\begin{abstract}
We study regular and black hole solutions to the coupled classical Einstein-Yang-MillsHiggs system. It has long been thought that black hole solutions in the spontaneously broken phase of such a theory could have no nontrivial field structure outside of the horizon. We first show that the standard black hole no-hair theorem underlying this belief, although true in the abelian setting, does not necessarily extend to the non-abelian case. This indicates the possibility of solutions with non-trivial gauge and Higgs configurations decaying exponentially outside the horizon. We then find such solutions by numerical integration of the classical equations for the case of $S U(2)$ coupled to a Higgs doublet (the standard model less hypercharge). As a prelude to this work we also study regular and black hole solutions to Einstein-Non-Abelian-Proca theory and as a postscript we briefly discuss the important issue of stability.
\end{abstract}




\section{Introduction}

Some time ago it was proven that black hole solutions in Einstein gravity coupled to the abelian Proca model or to a spontaneously broken abelian gauge theory cannot have any nontrivial field structure outside of the horizon [1-2]. In conjunction with similar arguments applied to a variety of field theories coupled to gravity [1-4], a widely held belief has been that the only distinguishing features of a black hole exterior to the horizon are charges carried by massless gauge fields [6]. These are mass, angular momentum and "electric" or "magnetic" charges, where the latter refer to charges carried by some possibly non-abelian gauge group. This belief is often referred to as the black hole no-hair theorem (or more precisely, no-hair conjecture).

In elementary particle physics we are familiar with the fact that non-abelian symmetries often play a crucial role. Based upon the abelian case and more general prejudice, it has generally been accepted that the no-hair statements ensure that no evidence of the broken classical charges can be found outside a black hole horizon. In particular, it has been thought that one cannot find stationary black hole solutions which are nonsingular at the event horizon and have a nonvanishing massive vector field that decays exponentially outside of the horizon (for example, see [1-2], [5] and [7-8]). It is the intent of the present paper to quantitatively investigate the veracity of this belief. That is, we study black hole solutions in Einstein gravity coupled to the non-abelian Proca model and to a spontaneously broken gauge theory (with symmetry group $S U(2)$ in each case) in order to determine whether the no-hair statements are affected by the non-abelian structure. Our results indicate that, in fact, the non-abelian nature of the theory has a crucial impact giving rise to classical solutions which violate the no-hair conjecture.

In particular, we numerically study spherically symmetric solutions to Einstein gravity coupled to $S U(2)$ gauge theory and a Higgs doublet, as in the standard model (without hypercharge). We take the standard model form for the symmetry breaking Higgs potential with only the lower real component of the Higgs field having a nonzero vacuum expectation value. Integrating via a two parameter shoot (associated with the initial values of the gauge and Higgs fields), we find black hole solutions in which there are nontrivial gauge and Higgs

fields outside of the horizon (with no global charges associated with the gauge or Higgs fields) that ultimately exponentially decay to their vacuum values. In fact, we find two families 
of such solutions with each member distinguished by the number $k$ of zero crossings of the single free function in the gauge connection. The $k=1$ member of one of these two families of solutions has a limit (the dimensionless Higgs vacuum expectation value going to zero) in which gravity becomes arbitrarily weak outside of the horizon and the solution resembles the known flat space sphaleron solution [9]. So, at least this particular solution might be characterized as a being a black hole with sphaleron hair. Our study indicates, however, that the other solutions do not have a weak gravity limit and hence do not have known flat space counterparts.

We note that ours is not the first challenge to the no-hair conjecture, but it is useful and important to emphasize the viewpoint espoused in [5]. As these authors point out, one should distinguish primary hair from secondary hair. The latter refers to black hole structures which exist solely as the result of (well known) primary hair such as gauge charges and hence are not fundamentally new characteristics. The existence of secondary hair, therefore, is not really in conflict with the no-hair conjecture. The charged dilaton black hole solutions [10-15] in which there is nontrivial scalar field configuration outside of the horizon provides an example of this distinction. The dilaton configuration is nontrivial because the electric charge (primary hair) acts as a source and hence yields only secondary hair. Another interesting solution which (among other things) provides a challenge to the no-hair theorems are the "black holes inside magnetic monopoles" found in [16, 17]. Again, however, the hair associated with the nontrivial field configuration outside of the horizon is of the secondary sort [5]. Two other challenges to the no-hair conjectures, which are closer to having primary hair, are the works of [18] (motivated by the paper of Bartnik and McKinnon [19]) and [20]. The work of [18] finds numerical black hole solutions of Einstein gravity coupled to $S U(2)$ gauge theory in which the fields decay sufficiently quickly so as to have vanishing global charges. The hair on these solutions is still carried by massless gauge fields - the novel feature is that the fields leave no imprint at infinity. Unfortunately, these solutions have been shown to be unstable [21]. In [20] some interesting numerical work also has shown that black holes can exist with nontrivial Skyrme fields in the Einstein-Skyrme model.

Our work on this subject was partly motivated by the publication of [19] in which the authors found smooth solutions to the coupled Einstein-SU(2) gauge theory classical equations [23]. These smooth solutions were surprising in that no-go theorems for classical 
glueball solutions to non-abelian gauge theory (without coupling to gravity) were established in [24] while no-go theorems for smooth solutions including gravity were proven for $2+1$ dimensions in [25]. Unfortunately, the $3+1$ dimensional solutions of [19] were subsequently proven to be unstable. A natural question to ask is whether such smooth solutions in $3+1$ dimensions can be found in the physically relevant case of spontaneously broken nonabelian gauge theories and, if so, whether they might be stable. In addition to our black hole solutions, we do in fact find and present such smooth solutions but have not as yet exhaustively analyzed their stability properties.

Stability is, in fact, an important unanswered question about both our black hole and regular solutions. However, we hasten to emphasize that the long held belief that such black hole solutions do not exist is based on the no-hair results which themselves have nothing to do with stability. Rather, the no-hair results were based on the careful examination of certain classical field theories coupled to gravity which simply have a different character from the non-abelian vector theories studied here. However, as far as physical relevance is concerned, stability is a crucial feature. General prejudice would certainly lean towards suspecting that our solutions are unstable for two main reasons. First, without a global charge leaving an imprint at infinity, stability certainly seems less likely. Second, as mentioned, there is a relation between our solutions and flat space sphalerons, the latter of which are unstable. On the other hand, some of our solutions seem to involve the trapping of gauge bosons in a region of space where, due to the Higgs configuration, they are less massive (relative to their asymptotic mass), and this, potentially, may bode well for stability. It has been noted that there might be a no-hair theorem if one only considers stable solutions [21]. We do not know, however, of any definitive arguments supporting this natural suggestion. We are presently studying the stability question for both the smooth and black hole solutions discussed here and will report on this elsewhere.

The organization of this paper is as follows. In section II we review the no-hair theorems and indicate why they do not apply in the situation under study. This opens up the possibility for the existence of black hole solutions with non-abelian gauge and Higgs hair. In section III we embark on the numerical construction of such solutions, beginning with the simpler Einstein-Non-Abelian-Proca system for the case of $S U(2)$. This system is interesting in its own right as a first concrete illustration of how the classical solitons in abelian and non-abelian Proca theories differ substantially. It also is a useful prelude to 
our discussion of the Einstein-Yang-Mills-Higgs system in section IV. In both sections III and IV we present spherically symmetric regular and black hole solutions. In section V we present some preliminary work on the stability and we offer our conclusions.

\section{Limitations on No-Hair Theorems}

Many of the no-hair proofs for classical field theories utilize an elegant and powerful method first developed by Bekenstein [1]. It is, in effect, a repackaging of the generally covariant field equations into a statement about the behavior of fields outside the event horizon. In this section, we briefly review Bekenstein's arguments and illustrate how nonabelian gauge fields in general, and the Einstein-Non-Abelian-Proca (ENAP) and EinsteinYang-Mills-Higgs (EYMH) systems in particular, can possess static black hole solutions with nontrivial exterior structure.

Consider the action for a set of arbitrary local fields $\Phi_{\mathrm{k}}$ in a gravitational background: $S=\int d^{4} x \widehat{\mathcal{L}} \equiv \int d^{4} x \sqrt{-\mathrm{g}} \mathcal{L}$. After multiplication by $d^{4} x \Phi_{\mathrm{k}}$ and integration by parts, the covariant Euler-Lagrange equations become

$$
\sum_{\mathrm{k}} \int_{\Omega} d^{4} x \partial_{\mu}\left[\Phi_{\mathrm{k}} \frac{\partial \widehat{\mathcal{L}}}{\partial\left(\Phi_{\mathrm{k}, \mu}\right)}\right]=\sum_{\mathrm{k}} \int_{\Omega} d^{4} x\left[\Phi_{\mathrm{k}, \mu} \frac{\partial \widehat{\mathcal{L}}}{\partial\left(\Phi_{\mathrm{k}, \mu}\right)}+\Phi_{\mathrm{k}} \frac{\partial \widehat{\mathcal{L}}}{\partial \Phi_{\mathrm{k}}}\right]
$$

The left-hand side of this equation can be expressed as a surface integral $\int_{\partial \Omega} d S_{\mu} b^{\mu}$, where $d S_{\mu}$ is an element of the hypersurface bounding the volume $\Omega$ over which we integrate, and

$$
b^{\mu} \equiv \sum_{\mathrm{k}} \Phi_{\mathrm{k}} \frac{\partial \widehat{\mathcal{L}}}{\partial\left(\Phi_{\mathrm{k}, \mu}\right)}
$$

If we assume that our system admits black hole solutions and we choose our four-volume to be the black hole exterior, then $\partial \Omega$ consists of the horizon, spatial infinity, and future and past timelike infinities. The behavior of $b^{\mu}$ at spatial infinity is already determined for all nontrivial and physically relevant fields by the field equations: $b^{\mu} \rightarrow 1 / r^{3}$ asymptotically for massless fields and $b^{\mu}$ vanishes exponentially for massive fields, so there is no contribution at spatial infinity to the left side of $(2.1)$. Since $d S_{\mu}$ is proportional to the normal $n_{\mu}$ of the hypersurface, which can be chosen to satisfy $n_{i}=0$ as $t \rightarrow \infty, d S_{\mu} b^{\mu}$ also vanishes 
at timelike infinity when $b^{0}=0$. This condition on $b^{0}$ is satisfied by static fields, and thus there are no non-horizon contributions to the surface integral in eq.(2.1). From the fact that the horizon is a null hypersurface $\left(g_{i j} d S^{i} d S^{j}=0\right)$ and that $g_{i j}$ is positive semi-definite on the horizon, it can easily be shown that $d S_{\mu} b^{\mu}$ vanishes on the horizon when $b_{\mu} b^{\mu}$ is bounded there. Thus, for static fields with finite $b_{\mu} b^{\mu}$ on the horizon, eq.(2.1) implies that the integral over the entire black hole exterior of some nontrivial function of the fields and their derivatives must vanish. The strategy for establishing no hair becomes clear: if one can demonstrate that the integrand in eq.(2.1) is negative or positive definite, then the only finite energy solutions satisfying (2.1) are those for which the integrand vanishes. For most Lagrangians of physical interest, this implies that the fields must assume constant values over the entire black hole exterior.

The Bekenstein approach has been used to establish no-hair theorems for Klein-Gordon theory, abelian Proca [1] and Higgs theories, and the Goldstone model [2], among others. Here we provide a sketch of the right-hand side of eqn.(2.1) for non-abelian gauge fields which demonstrates the possibility of the existence of Einstein-Yang-Mills (EYM) black holes.

The Lagrangian for an $S U(2)$ gauge theory may be written

$$
\mathcal{L}_{\mathrm{EYM}}=-\frac{1}{4 \pi}\left(\frac{1}{4}|F|^{2}\right) \equiv-\frac{1}{4 \pi}\left(\frac{1}{4} g^{\mu \rho} g^{\nu \sigma} F_{\mu \nu}^{(i)} F_{\rho \sigma}^{(i)}\right)
$$

where $i$ is the isospin index. A straightforward calculation of the right side of eq.(2.1) for a metric with signature $(-1,+1,+1,+1)$ gives

$$
-\frac{1}{4 \pi} \int_{\Omega} d^{4} x \sqrt{-\mathrm{g}}\left(-8 \pi \mathcal{L}_{\mathrm{EYM}}+\frac{1}{2}\left[g \epsilon_{i j k} A_{\mu}^{(j)} A_{\nu}^{(k)}\right] F^{(i) \mu \nu}\right),
$$

where $g$ is the gauge coupling: $F_{\mu \nu}^{(i)}=\partial_{\mu} A_{\nu}^{(i)}-\partial_{\nu} A_{\mu}^{(i)}+g \epsilon_{i j k} A_{\mu}^{(j)} A_{\nu}^{(k)}$. If we consider only static fields, and for simplicity assume $A_{0}^{(i)}=0$, then $|F|^{2} \geq 0$. The second term in the integrand, however, is not necessarily positive: its sign will depend on the details of the solution in the black hole exterior $\Omega$, so it is clear that the nonlinear terms can lead to a possible way of avoiding this no-hair argument.

To demonstrate this point more quantitatively, consider the expression in (2.4). Let $C_{\mu, \nu}^{(i)}=\partial_{\mu} A_{\nu}^{(i)}-\partial_{\nu} A_{\mu}^{(i)}$ and $D_{\mu, \nu}^{(i)}=g \epsilon_{j k}^{i} A_{\mu}^{(j)} A_{\nu}^{(k)}$. Then to avoid the Bekenstein argument 
we require the non-positivity (somewhere) of $S \equiv(C+D) \cdot(C+D)+D \cdot(C+D)$ where $C \cdot C=C_{\mu, \nu}^{(i)} C^{(i) \mu \nu}$ etc. With the gauge choice $A_{0}^{(i)}=0$, and the assumption that in this gauge all fields are time independent (no 'electric fields'), it follows that $(\alpha C+\beta D) \cdot(\alpha C+\beta D) \geq 0$ for any real $\alpha, \beta$. The relation $S=(C+2 D) \cdot(C+D) \leq 0$ requires that $C \cdot D \leq 0$, and gives $C \cdot C+2 D \cdot D \leq-3 C \cdot D$. This may be rephrased as

$$
\frac{1}{2}(C \cdot C+D \cdot D)-\frac{1}{6}(C \cdot C-D \cdot D) \leq-C \cdot D \leq \frac{1}{2}(C \cdot C+D \cdot D),
$$

where the second inequality is just the positivity of $(C+D) \cdot(C+D)$.

Noting that $D$ is the non-abelian term we see that the non-abelian nature of the field is crucial in avoiding the Bekenstein argument for no-hair. The above relation also shows that the value of $C \cdot D$ must lie in a very restricted range for the non-positivity of $S$ : the cubic term in the fields involving the structure constants is bounded both above and below by the quadratic and quartic terms in the gauge fields. (It is possible to make restricted gauge transformations that preserve time independence of fields and $A_{0}^{(i)}=0$. What we see is that (2.5) must hold in some region in each such gauge.)

The left-hand side of eq.(2.1) also presents complications. For a gauge field, $b_{\mu} b^{\mu}$ is not a physical scalar and need not be bounded on the horizon, so a practical application of the Bekenstein approach requires the explicit evaluation of

$$
b^{\mu}=-\frac{1}{4 \pi} F^{(i) \mu \nu} A_{\nu}^{(i)}
$$

on the horizon to make the sign considerations for eq.(2.4) relevant. By introducing the ansatz for the connection $A$ used in [19] and [18], it can be shown that $b_{\mu} b^{\mu}$ is bounded on the horizon and that the second term in (2.4) can be negative in the black hole exterior, so that a black hole with nontrivial gauge field structure outside the horizon is allowable (but not necessary) under the Bekenstein analysis. In fact, the solutions of [18] are examples of these "colored" black holes.

When this approach is applied to the ENAP theory considered below, the results are similar. For our metric convention, the Lagrangian is

$$
\mathcal{L}_{\mathrm{ENAP}}=-\frac{1}{4 \pi}\left(\frac{1}{4}|F|^{2}+\frac{\mu^{2}}{2}|A|^{2}\right)
$$

with $|A|^{2} \equiv g^{\mu \nu} A_{\mu}^{(i)} A_{\nu}^{(i)}$ and $\mu$ is the vector field mass. As we stated above, this is obviously 
not a gauge-invariant theory but provides a good first approximation for a massive nonabelian gauge field. For the massive field, $b^{\mu} b_{\mu}$ is now a physical scalar bounded on the horizon, and the Bekenstein integral (2.1) assumes the same form as in (2.4), with $\mathcal{L}_{\mathrm{EYM}}$ replaced by $\mathcal{L}_{\text {ENAP. }}$ For $A_{0}^{(i)}=0$, the same arguments as above establish the possibility of hair for massive vector black hole solutions.

The second theory we investigate in this paper is EYMH, whose Lagrangian is given by

$$
\mathcal{L}_{\mathrm{EYMH}}=\mathcal{L}_{\mathrm{EYM}}-\frac{1}{4 \pi}\left[\left(D_{\mu} \Phi\right)^{\dagger}\left(D^{\mu} \Phi\right)+V(\Phi)\right]
$$

where $D_{\mu}=\partial_{\mu}+g \tau \cdot \mathbf{A}_{\mu}$ is the usual gauge-covariant derivative expressed in the antihermitian representation of $s u(2) \quad\left(\tau_{i}=-i \sigma_{i} / 2\right)$. The Higgs field $\Phi$ is taken to be a complex doublet $\Phi=\left(\phi^{+}, \phi^{-}\right)$and $V(\Phi)$ to be a double-well potential with degenerate vacua. Though we give further details of our Higgs ansatz below, for now we assume $\Phi$ possesses only one degree of freedom:

$$
\Phi=\frac{1}{\sqrt{2}}\left(\begin{array}{c}
0 \\
\phi(\mathbf{x})
\end{array}\right)
$$

for $\phi$ real and time-independent. The Lagrangian (2.8) then becomes

$$
\mathcal{L}_{\mathrm{EYMH}}=-\frac{1}{4 \pi}\left(\frac{1}{4}|F|^{2}+\frac{1}{2}\left(\frac{g \phi}{2}\right)^{2}|A|^{2}+\frac{1}{2}|\partial \phi|^{2}+V(\phi)\right),
$$

where $|\partial \phi|^{2} \equiv g^{\mu \nu} \partial_{\mu} \phi \partial_{\nu} \phi \geq 0$, and $(g \phi / 2)$ now plays the role of the gauge field mass. The vector $b^{\mu}$ has an additional term

$$
b^{\mu}=-\frac{1}{4 \pi}\left[F^{(i) \mu \nu} A_{\nu}^{(i)}+g^{\mu \nu}\left(\partial_{\nu} \phi\right) \phi\right]
$$

and the Bekenstein integral is

$$
\begin{gathered}
-\frac{1}{4 \pi} \int_{\Omega} d^{4} x \sqrt{-\mathrm{g}}\left[\frac{1}{2}|F|^{2}+\left(\frac{g \phi}{2}\right)^{2}|A|^{2}+\frac{1}{2}\left[g \epsilon_{i j k} A_{\mu}^{(j)} A_{\nu}^{(k)}\right] F^{(i) \mu \nu}\right] \\
-\frac{1}{4 \pi} \int_{\Omega} d^{4} x \sqrt{-\mathrm{g}}\left[|\partial \phi|^{2}+\left(\frac{g \phi}{2}\right)^{2}|A|^{2}+V^{\prime}(\phi) \phi\right],
\end{gathered}
$$

with $V^{\prime}(\phi) \equiv d V(\phi) / d \phi$. The separate integrals in this expression correspond to the YM and Higgs surface integrals obtained from eq.(2.11). The YM term is identical in form to the 
Proca case, so by the above arguments YM hair is possible. In the Higgs term, the sign of $V^{\prime}(\phi) \phi$ for a double-well $V(\phi)$ obviously depends on the details of a solution, so the second integrand need not be positive definite either. Black hole solutions to EYMH theory having both nontrivial YM and Higgs field structure are therefore not excluded by the Bekenstein analysis.

It is important to emphasize that these sign arguments only demonstrate the possibility of solutions with hair. An example for which all of the above reasoning is valid but a no-hair proof has been found [2] is the pure Higgs (Goldstone) theory, which corresponds to the Higgs contribution to $(2.12)$ with $|A|^{2} \equiv 0$. The Higgs-gauge field coupling and the nonabelian gauge interactions in EYMH theory therefore play a critical role in the existence of black hole solutions for which $\phi \not \equiv$ constant outside the horizon. We should also mention that Price [4] has employed somewhat different reasoning to argue for a black hole no-hair theorem. His arguments, though, are only valid in the domain of weak fields (in which the important nonlinear terms would lose their influence) and hence do not directly apply to the present context. In the following we shall show that the gap in the no-hair arguments described is wide enough to allow for solutions which have spontaneously broken gauge and Higgs hair.

\section{ENAP Theory: Regular and Black Hole Solutions}

\section{Metric and Connection}

The metric for a static, spherically symmetric spacetime can be written

$$
d s^{2}=-T^{-2}(r) d t^{2}+R^{2}(r) d r^{2}+r^{2}\left(d \theta^{2}+\sin ^{2} \theta d \varphi^{2}\right)
$$

where $R(r) \equiv(1-2 m / r)^{-1 / 2}$ and $m(r)$ may be interpreted as the total mass-energy within the radius $r$. An alternate form of the metric convenient for describing black hole solutions follows from defining $\delta \equiv-\ln (R / T)$. In terms of the functions $(\delta(r), m(r))$, the metric becomes

$$
d s^{2}=-\left(1-\frac{2 m}{r}\right) e^{-2 \delta} d t^{2}+\left(1-\frac{2 m}{r}\right)^{-1} d r^{2}+r^{2}\left(d \theta^{2}+\sin ^{2} \theta d \varphi^{2}\right) .
$$

For the metric (3.1), regularity at the origin requires $T(0)<\infty$ and $R^{\prime}(r), T^{\prime}(r) \rightarrow 0$, while asymptotic flatness requires $R(r), T(r) \rightarrow 1$. For the alternate form of the metric, a 
regular event horizon at $r=r_{h}$ requires

$$
m\left(r_{h}\right)=r_{h} / 2, \quad \delta\left(r_{h}\right)<\infty
$$

and comparison with the asymptotics of eq.(3.2) gives $\delta(r) \rightarrow 0$ as $r \rightarrow \infty$. For static solutions, however, we can rescale the time coordinates

$$
\begin{aligned}
d \widetilde{t} & \equiv T^{-1}(0) d t \\
d \widetilde{t} & \equiv e^{-\delta\left(r_{h}\right)} d t
\end{aligned}
$$

in order to simplify the initial data problem: for such a rescaling,

$$
\begin{aligned}
\widetilde{T}(0) & =1, & & \widetilde{T}(\infty)=1 / T_{o} \equiv 1 / T(0) \\
\widetilde{\delta}\left(r_{h}\right) & =0, & & \widetilde{\delta}(\infty)=-\delta_{o} \equiv-\delta(0) .
\end{aligned}
$$

Thus the correct initial values of $g_{t t}$ for a given system can be determined by integrating the Einstein equations with the fully specified initial conditions

$$
\begin{aligned}
R(0)=1, & T(0)=1 \\
m\left(r_{h}\right)=r_{h} / 2, & \delta\left(r_{h}\right)=0
\end{aligned}
$$

where in each of the last three equations, the top part refers to regular solutions and the bottom part to black hole solutions. We will use this parameterization below.

The most general spherically symmetric $S U(2)$ connection [26] is

$$
A=\frac{1}{g}\left[a \widehat{\tau}_{r} d t+b \widehat{\tau}_{r} d r+\left(d \widehat{\tau}_{\theta}-(1+c) \widehat{\tau}_{\varphi}\right) d \theta+\left((1+c) \widehat{\tau}_{\theta}+d \widehat{\tau}_{\varphi}\right) \sin \theta d \varphi\right],
$$

where $a$ and $b$ have dimensions $[L]^{-1}, c$ and $d$ are dimensionless, $g$ is the gauge coupling and $\left(\widehat{\tau}_{r}, \widehat{\tau}_{\theta}, \widehat{\tau}_{\varphi}\right)$ is the antihermitian $s u(2)$ basis expressed in the usual 3-d (physical space) polar coordinate directions; e.g. $\widehat{\tau}_{r}=\widehat{\mathbf{r}} \cdot \tau$, and $\left[\widehat{\tau}_{a}, \widehat{\tau}_{b}\right]=\epsilon_{a b c} \widehat{\tau}_{c}$ with the indices ranging over $(r, \theta, \varphi)$. The four degrees of freedom $a, b, c, d$ are all functions of the 3 -d radius $r$ and time $t$. The connection (3.7) has a residual gauge freedom

$$
A \longrightarrow U A U^{-1}+\frac{1}{g} U d U^{-1}
$$

under unitary transformations of the form $U=\exp \left[\beta(r, t) \widehat{\tau}_{r}\right]$, where $\beta(r, t)$ is an arbitrary real function. Under such gauge transformations, which form an abelian subgroup of the full 
gauge group, the connection functions transform as

$$
U:\left(\begin{array}{l}
a \\
b \\
c \\
d
\end{array}\right) \longmapsto\left(\begin{array}{c}
\widetilde{a} \\
\widetilde{b} \\
\widetilde{c} \\
\widetilde{d}
\end{array}\right)=\left(\begin{array}{c}
a-\dot{\beta} \\
b-\beta^{\prime} \\
c \cos \beta-d \sin \beta \\
d \cos \beta+c \sin \beta
\end{array}\right) .
$$

We can use this freedom to impose the "polar gauge" $\widetilde{b} \equiv 0$. In the static case, $\dot{\beta}=0$ and $\widetilde{a}, \widetilde{c}, \widetilde{d}$ are functions of $r$ only. Following [19] and [18], we eliminate two of the three remaining degrees of freedom by the ansatz $\widetilde{a} \equiv 0$ (no dyons, purely magnetic YM curvature) and $\widetilde{d} \equiv 0$.

After relabelling the remaining degree of freedom $w(r) \equiv \widetilde{c}(r)$, the connection assumes the form

$$
A=\frac{1}{g}(1+w)\left[-\widehat{\tau}_{\varphi} d \theta+\widehat{\tau}_{\theta} \sin \theta d \varphi\right]
$$

which was explored by t'Hooft in the context of magnetic monopoles [28]. It differs from the ansatz of [19] by a singular gauge transformation $U=\exp \left(\theta \tau_{1}\right) \exp \left(\left(\frac{\pi}{2}-\varphi\right) \tau_{3}\right)$ and has the added virtue that $|A|^{2}$ is invariant under spatial rotations.

\section{ENAP Equations and Boundary Conditions}

Defining $F=d A+g A \wedge A$, we have

$$
F=\frac{w^{\prime}}{g}\left[-\widehat{\tau}_{\varphi} d r \wedge d \theta+\widehat{\tau}_{\theta} \sin \theta d r \wedge d \varphi\right]-\frac{1}{g}\left(1-w^{2}\right) \widehat{\tau}_{r} \sin \theta d \theta \wedge d \varphi
$$

The Proca equations $D^{*} F+\mu^{2 *} A=0$ reduce to a single equation:

$$
\frac{d}{d r}\left(\frac{w^{\prime}}{R T}\right)+\frac{w\left(1-w^{2}\right)}{r^{2}} \frac{R}{T}-\mu^{2}(1+w) \frac{R}{T}=0
$$

The Einstein equations $G_{\mu \nu}=8 \pi T_{\mu \nu}$ may be calculated by varying with respect to $g_{\mu \nu}$ the action

$$
\int_{\Omega} d^{4} x \sqrt{-\mathrm{g}}\left(\frac{R}{16 \pi}+\mathcal{L}_{\mathrm{ENAP}}\right)=\frac{1}{16 \pi} \int_{\Omega} d^{4} x \sqrt{-\mathrm{g}}\left(R-|F|^{2}-2 \mu^{2}|A|^{2}\right),
$$

where $R=R_{\mu \nu} g^{\mu \nu}$ is the gravitational curvature scalar and we have set $G=1$. The 
energy-momentum tensor is

$$
8 \pi T_{\mu \nu}=2 F_{\mu \gamma} F_{\nu}^{\gamma}-\frac{1}{2} g_{\mu \nu}|F|^{2}+\left[2 \mu^{2} A_{\mu} A_{\nu}-g_{\mu \nu} \mu^{2}|A|^{2}\right]
$$

and the $(t t)$ and $(r r)$ Einstein equations can be written

$$
\begin{aligned}
& m^{\prime}=\left(1-\frac{2 m}{r}\right)\left(\frac{w^{\prime}}{g}\right)^{2}+ \frac{1}{2} \frac{\left(1-w^{2}\right)^{2}}{g^{2} r^{2}}+\frac{\mu^{2}}{g^{2}}(1+w)^{2} \\
& r\left(1-\frac{2 m}{r}\right) \frac{T^{\prime}}{T}=-\left(1-\frac{2 m}{r}\right)\left(\frac{w^{\prime}}{g}\right)^{2}+\frac{1}{2} \frac{\left(1-w^{2}\right)^{2}}{g^{2} r^{2}} \\
&+\frac{\mu^{2}}{g^{2}}(1+w)^{2}-\frac{m}{r}
\end{aligned}
$$

in terms of the metric functions $m(r)$ and $T(r)$. While (3.16) is appropriate for obtaining regular solutions, the coordinate singularity at the event horizon of a black hole solution leads us to replace it with

$$
\delta^{\prime}=-\frac{2\left(w^{\prime}\right)^{2}}{g^{2} r},
$$

where $\delta$ was introduced in (3.2) above. For either choice of metric functions, the two Einstein equations can be used to express the Proca equation (3.12) in a form independent of $T(r)$ or $\delta(r)$ :

$$
\begin{aligned}
r^{2}\left(1-\frac{2 m}{r}\right) w^{\prime \prime}+\left[2 m-\frac{\left(1-w^{2}\right)^{2}}{g^{2} r}\right. & \left.-2 \frac{\mu^{2}}{g^{2}}(1+w)^{2} r\right] w^{\prime} \\
& +\left(1-w^{2}\right) w-\mu^{2}(1+w) r^{2}=0 .
\end{aligned}
$$

All of the above agrees with the corresponding EYM results $[19,18]$ when we take $\mu=0$.

The presence of the additional parameter $\mu$ in ENAP theory motivates us to clarify the role of the gauge coupling $g$ in the field equations. A classical theory with $G=c=1$ satisfies $[L]=[T]=[M]$, which for our theory implies $[g]=[\mu]=[L]^{-1}$. If we introduce the 
dimensionless quantities

$$
\widehat{r} \equiv g r, \quad \widehat{m}(\widehat{r}) \equiv g m(g r), \quad \widehat{\mu} \equiv \mu / g,
$$

then the theory scales as

$$
|F|^{2}=g^{2}|F(\widehat{r})|^{2}, \quad|A|^{2}=|A(\widehat{r})|^{2}, \quad \mathcal{L}_{\mathrm{ENAP}}=g^{2} \mathcal{L}_{\mathrm{ENAP}}(\widehat{r}, \widehat{\mu}),
$$

and the dimensionless ENAP equations assume the form of (3.15)-(3.18) with $g=1$ and (3.19) replacing the dimensionful variables and parameters. Through scaling, we may therefore obtain a solution to the ENAP equations for any $g>0$ from a solution to the dimensionless equations:

$$
\begin{aligned}
w_{g}(r) & =\widehat{W}(g r) & R_{g}(r)=(1-2 \widehat{m}(g r) /(g r))^{-1 / 2} \\
m_{g}(r) & =\frac{1}{g} \widehat{m}(g r) & T_{g}(r)=T(g r) ; \delta_{g}(r)=\delta(g r) .
\end{aligned}
$$

Solutions with $g>1$, for example, possess the same structure as $g=1$ solutions, but it occurs at radius $r=\widehat{r} / g<\widehat{r}$. For the remainder of this section, we take $g=1$ without loss of generality.

We can anticipate from the field equations the general characteristics of solutions. From the definition of $\delta$, eq.(3.17) demonstrates that $R / T$ increases monotonically with radius. $T$ can also be shown to satisfy the same equation as in EYM theory [19],

$$
\frac{d}{d r}\left[\frac{r^{2}}{R}\left(\frac{1}{T}\right)^{\prime}\right]=2\left(1-\frac{2 m}{r}\right)\left(w^{\prime}\right)^{2} \frac{R}{T}+\left(\frac{R}{r^{2} T}\right)\left(1-w^{2}\right)^{2},
$$

so that $T^{\prime}<0$ for $w \not \equiv 1$. From the boundary conditions of the unrescaled metric $(T(0)>$ $R(0)=1, T(\infty)=R(\infty)=1)$ and the definition of $R$, we then expect that $T>R \geq 1$, $R$ should possess at least one maximum, and $T$ should decrease monotonically for nontrivial regular solutions. For black hole solutions, $\delta$ should decrease monotonically and $R$ should decrease from its singular value at the horizon, though it need not possess any local extrema for $r_{h}<r<\infty$. 
Following [19], we can learn about the behavior of the vector field by rewriting the Proca equation in the form

$$
\frac{1}{2} \frac{d}{d r}\left[\frac{\left(w^{2}\right)^{\prime}}{R T}\right]=\frac{\left(w^{\prime}\right)^{2}}{R T}+\frac{R}{r^{2} T}\left[\left(w^{2}-1\right) w^{2}+\mu^{2}(1+w) w r^{2}\right]
$$

A trivial $m=0$ solution occurs for $w \equiv-1$, and comparison with eq.(3.15) reveals that $w=-1$ is the only acceptable asymptotic value for finite energy solutions. Since the righthand side of this equation is manifestly positive for $w^{2}>1$, the only nontrivial solutions having finite $w^{2}$ and finite energy must satisfy $w^{2} \leq 1$. The precise finite energy restrictions on the behavior of $w$ come from the nonderivative term in (3.23), and since $w^{\prime}=0$ gives the relation

$$
w w^{\prime \prime}=\frac{R^{2}}{r^{2}}(1+w) w\left[w(w-1)+\mu^{2} r^{2}\right] \quad\left(w^{\prime}=0\right)
$$

this term also governs the oscillatory properties of finite energy solutions. From (3.24), we see that $w^{\prime \prime}>0$ for $r>1 / 2 \mu$, so finite energy solutions must have their final turning points before $r=1 / 2 \mu$ and satisfy $w^{\prime}<0$ after $r=1 / 2 \mu$. For $r<1 / 2 \mu$, on the other hand, eqn.(3.24) indicates that $w w^{\prime \prime}<0$ in the range $\left(1-\sqrt{1-(2 \mu r)^{2}}\right) / 2<w<$ $\left(1+\sqrt{1-(2 \mu r)^{2}}\right) / 2$, so solutions can exhibit nontrivial behavior for $w>0$ and still satisfy the boundary conditions at infinity. If $w>\left(1+\sqrt{1-(2 \mu r)^{2}}\right) / 2$, however, w will increase beyond $w^{2}=1$, so this range is forbidden for finite energy solutions. Since we have used only the $r \rightarrow \infty$ boundary conditions and not mentioned initial conditions on $w$ or the metric in this discussion, the results

$$
\begin{aligned}
& r<\frac{1}{2 \mu}: \quad-1<w<\frac{1}{2}\left(1+\sqrt{1-(2 \mu r)^{2}}\right) \\
& r>\frac{1}{2 \mu}: \quad w^{\prime}<0, \quad w^{\prime \prime}>0
\end{aligned}
$$

describe both regular and black hole solutions.

The full boundary conditions may be determined from eqs.(3.15)-(3.18). For regular solutions, finite energy density $T_{t t}$ and regularity of $T(r)$ at the origin $\left(T^{\prime}(0)=0\right)$ give 


$$
\begin{aligned}
2 m(r) & =\mathcal{O}\left(r^{3}\right) \\
\ln T(r) & =\mathcal{O}\left(r^{2}\right) \\
w(r) & =-1+\mathcal{O}\left(r^{2}\right) .
\end{aligned}
$$

It is significant that $w=-1$ is the only possible initial value; in EYM theory the selfinteraction term $\left(1-w^{2}\right)^{2} / r^{2}$ in $m^{\prime}$ provides the two possibilities $w_{ \pm}= \pm 1$ [19], but the vector field mass in our theory excludes $w_{+}$both at the origin and as $r \rightarrow \infty$. The $\mu^{2}$ terms also breaks the discrete symmetry $w \rightarrow-w$ of the EYM equations which gives rise to degenerate mirror-image solutions in [19] and [18]. These differences will play an important role in the spectrum of solutions.

For black hole solutions, the field equations with $m\left(r_{h}\right)=r_{h} / 2$ give $m^{\prime}\left(r_{h}\right), w^{\prime}\left(r_{h}\right)$, and $\delta^{\prime}\left(r_{h}\right)$ when $\mu$ is specified. Full use of the metric initial conditions allows us to expand near the horizon:

$$
\begin{aligned}
m(r) & =r_{h} / 2+m^{\prime}\left(r_{h}\right)\left(r-r_{h}\right)+\mathcal{O}\left(r-r_{h}\right)^{2} \\
\delta(r) & =0+\delta^{\prime}\left(r_{h}\right)\left(r-r_{h}\right)+\mathcal{O}\left(r-r_{h}\right)^{2} \\
w(r) & =w\left(r_{h}\right)+w^{\prime}\left(r_{h}\right)\left(r-r_{h}\right)+\mathcal{O}\left(r-r_{h}\right)^{2},
\end{aligned}
$$

where $r_{h}$ and $w\left(r_{h}\right)$ are to be chosen to yield a finite energy solution.

Since the only allowable vacuum value of the connection function for both regular and black hole solutions is $w=-1$, the behavior of eqs.(3.15)-(3.18) as $r \rightarrow \infty$ gives

$$
\begin{aligned}
m(r) & \sim M-\mu c^{2} e^{-2 \mu r} \\
\ln T(r) & \sim \ln \left(\frac{1}{T_{o}}\right)+\frac{M}{r} \\
\delta(r) & \sim-\delta_{o}+\mu c^{2} \frac{e^{-2 \mu r}}{r}, \\
w(r) & \sim-1+c e^{-\mu r},
\end{aligned}
$$

where $c$ is some positive constant and $T_{o}$ and $\delta_{o}$ refer to the unrescaled initial values introduced in eqs.(3.5). Thus the presence of the $\mu^{2}$ terms in ENAP theory give exponentially, rather than polynomially, decaying fields as we expect. 


\section{Numerical Regular Solutions}

We use a standard one-parameter "shooting" method to find regular solutions to the ENAP equations. The formal power series describing the boundary conditions (3.26)(3.28) at $r=0$ is

$$
\begin{aligned}
2 m(r) & =4 b^{2} r^{3}+\frac{2}{5}\left(-8 b^{3}+3 b^{2} \mu^{2}\right) r^{5}+\mathcal{O}\left(r^{7}\right) \\
\ln T(r) & =-2 b^{2} r^{2}-\frac{1}{5}\left(12 b^{4}-4 b^{3}+\mu^{2} b^{2}\right) r^{4}+\mathcal{O}\left(r^{6}\right) \\
w(r) & =-1+b r^{2}+\frac{1}{10}\left(8 b^{3}-3 b^{2}+\mu^{2} b\right) r^{4}+\mathcal{O}\left(r^{6}\right),
\end{aligned}
$$

which depend only on $b>0$ and $\mu$ and conform to the EYM results [19] for $\mu=0$. We use these conditions evaluated at $r=10^{-3}$ as initial data in a standard ordinary differential equation solver with a global error tolerance of $10^{-12}$, adjusting $b$ for fixed $\mu$ and integrating outward in an attempt to meet the boundary conditions at infinity. The bracketing condition for finite energy solutions is the same as in [19]: for a small range of $b$ in the vicinity of a solution, $w$ approaches its asymptotic value $w \rightarrow-1$ and either crosses through $w=-1$ and rapidly goes to $w=-\infty$, or experiences a turning point and rapidly goes to $w=+\infty$. Though $2 m(r) \rightarrow r$ as $|w|$ diverges, at a discrete value of $b$ in this range lies a finite energy regular solution with the correct asymptotics. The existence of such solutions has been proven rigorously only in the $\mu=0$ case [22], but the qualitatively similar numerical behavior for $\mu>0$ lends strong support for our work.

As in EYM theory, finite energy solutions are characterized by the oscillatory behavior anticipated above in the near-field region $r \gtrsim 1$. Distinct solutions are classified by $k$, the number of zeros of $w$, which increases with increasing $b$ but must be even for $\mu>0$ to conform to the boundary conditions (3.35) and (3.38). A striking difference between our non-abelian Proca solutions and the EYM solutions of [19] is that for $\mu>0$, we find two distinct classes of even- $k$ solutions. We will now discuss each class in turn.

One class possesses physical characteristics very similar to the even- $k$ solutions of [19] for small $\mu$, reducing to them in the limit $\mu \rightarrow 0$; the presence of the vector field mass only slightly perturbs the gross behavior of the field. Because it offers the best hope for dynamical stability, we focus our attention on the $k=2$ solutions. In the range $0<\mu<4.454 \times 10^{-2}$, the shooting parameter varies over $0.6517>b>0.5787$ while the width of the single peak of $w(r)$ 
increases as the mass increases: $0.9713<M<0.9936$ (cf. fig. 1a). For $\mu>4.454 \times 10^{-2}$, $w$ does not approach its asymptotic value before an additional turning point occurs and $w \rightarrow+\infty$ : the $\mu^{2}$ term in the Proca equation begins to dominate at a radius $r \sim 1 / \mu \approx 20$ before the non-abelian self-interaction becomes negligible, thereby making impossible $k$-node solutions for any value of $b$. Another way to understand the occurrence of a maximum $\mu$ value is through eqn.(3.15); if the $\mu^{2}$ term dominates the energy density, the radius will always approach the Schwarzschild radius $r=2 m$ beyond some value of $\mu$ and solutions with smooth geometry will be forbidden.

The second class of solutions resembles the $(k-1)$ odd-node solutions of [19] as $\mu \rightarrow 0$, with $w$ approaching the forbidden asymptotic value $w=+1$ until large $r<1 / 2 \mu$, where a turning point and then and additional node occur before $w \rightarrow-1$ (fig. 1b). As the mass parameter increases toward $\mu=4.454 \times 10^{-2}$, this type of solution begins to resemble the limiting case of the first solution type, until eventually the two classes converge at the maximum value of $\mu$ : for $0<\mu<4.454 \times 10^{-2}$, the shooting parameter and total mass vary over $0.4537<b<0.5787$ and $0.8286<M<0.9936$, respectively. Thus solutions to ENAP theory bifurcate into two branches, with the bifurcation point corresponding to the $\mu$ at which $k$-noded solutions no longer occur. In shooting parameter space, the bifurcation condition corresponds to the shrinking of the interval over which $k$ nodes occurs from the maximal value of [19] $(0.4537<b<0.6517$ for $k=2)$ to zero, with finite-energy solutions occurring at the endpoints.

The existence of a second class of solutions and the bifurcation phenomenon can be understood from a heuristic argument of scales. In EYM theory, there is one dimensionful parameter $g$ whose units are $[T][M]^{-1 / 2}[L]^{-3 / 2}$ (where for the remainder of this paragraph we do not set $G=1$ ) which sets the scale of the solutions to be on the order of $G^{1 / 2} g^{-1}$. By scale here we refer to the approximate value of the radius beyond which the fields in the theory exhibit no nontrivial behavior and rapidly approach their asymptotic values. In ENAP theory, on the other hand, we have two dimensionful parameters $[g]$ and $[\mu]$, the latter of whose units is $[L]^{-1}$. There are thus two distinct length scales : $G^{1 / 2} g^{-1}$ and $\mu^{-1}$. The former sets the scale for one class of our solutions and the latter sets the scale for our second class. Notice that the first scale is dependent on gravitational interactions while the second is not. As $1 / \mu \rightarrow \infty$, the last node of a $k$-node solution in the second family is pushed off to infinity and the solution approaches an odd-node solution of the EYM system with 
its scale being set by the only remaining parameter, $G^{1 / 2} g^{-1}$. These quasi-odd- $k$ solutions [29] therefore have the scale of their inner structure set by gravity while the scale of their asymptotic structure is set by the Proca mass [31].

\section{Numerical Black Hole Solutions}

To find numerical black hole solutions, we followed a similar shooting procedure. The field equations give

$$
\begin{aligned}
m^{\prime}\left(r_{h}\right) & =\left[1-w^{2}\left(r_{h}\right)\right]^{2} / 2 r_{h}^{2}+\mu^{2}\left[1+w\left(r_{h}\right)\right]^{2} \\
w^{\prime}\left(r_{h}\right) & =\frac{\mu^{2}\left[1+w\left(r_{h}\right)\right] r_{h}^{2}-\left[1-w^{2}\left(r_{h}\right)\right] w\left(r_{h}\right)}{r_{h}-\left[1-w^{2}\left(r_{h}\right)\right]^{2} / r_{h}-2 \mu^{2}\left[1+w\left(r_{h}\right)\right]^{2} r_{h}} \\
\delta^{\prime}\left(r_{h}\right) & =-2\left[w^{\prime}\left(r_{h}\right)\right]^{2} / r_{h}
\end{aligned}
$$

on the horizon, and we can construct initial data from eqs.(3.29)-(3.30) with only the shooting parameter $w\left(r_{h}\right)$ and the horizon radius $r_{h}$ unspecified. Following [18], we examine $r_{h}=1$ and chose $\left(r-r_{h}\right)<10^{-12}$ so that the errors in the initial data are smaller than the global tolerance.

As in the smooth case, we again find two distinct classes of solutions whose scales are respectively set by the two dimensionful parameters in the theory. In appropriate limits, these solutions approach those of [18] modulo one interesting special case to be mentioned below. For $\mu>0$ we find that odd-node solutions are possible in addition to even- $k$ solutions, since the horizon shields the singularity which would occur at $w(0)=+1$ for such regular solutions. These $k$-node solutions are classifiable by their behavior as $\mu \rightarrow 0$; one class reduces to the $k$-node solutions of [18], while the other approaches the $(k-1)$-node solutions of [18] as the position of the $k$ th node moves out to $r=\infty$. The two solution branches are again joined at a bifurcation point for some maximum value of $\mu$ for each $k$. The $k=1$ quasi-even-node case is special because there exists no corresponding non-abelian solution with $k-1=0$ nodes in [18]; instead, this solution approaches $w \equiv+1$ as $\mu \rightarrow 0$, which corresponds to the ordinary Schwarzschild solution. The (regular and black hole) analogue of this solution in Einstein-Yang-Mills-Higgs theory (to be discussed shortly) is significant as it allows us to make contact with the known existence of a flat space sphaleron. This is the only set of solutions (black hole or regular) for which the limiting case is essentially weak-gravity (weak in the present sense meaning that it becomes arbitrarily close to Schwarzschild). Hence, 
ENAP black holes are a set of fundamentally non-abelian solutions which, like the solutions of [18], possess metrics which interpolate between the RN and Schwarzschild metrics, but which include as limiting cases both quasi-Schwarzschild and quasi-RN $(k \rightarrow \infty$ here and in [18]) solutions. In this sense, the spectrum of solutions itself interpolates between the Schwarzschild and pure-magnetic RN black holes.

Though the solutions for all node numbers exhibit bifurcation, we focus our attention on the lowest odd- and even-node solutions. In figs. $2 \mathrm{a}-\mathrm{b}$, the mass and connections for the two branches of the $k=1$ solutions are plotted. The limiting value of $\mu=0.1233$ joins the solution branches, which are described by $1>w\left(r_{h}\right)>0.8500,0.5000<M<$ 1.0052, $0<\delta_{o}<0.2854$ (quasi- $k=0$ ) and $0.6322<w\left(r_{h}\right)<0.8500,0.9372<M<$ $1.0052,0.5485>\delta_{o}>0.2854$. For $k=2$, the maximum value $\mu=1.752 \times 10^{-2}$ gives the branches $-0.6322<w\left(r_{h}\right)<-0.5027,0.9372<M<1.0052,0.5485<\delta_{o}<0.5831$ (quasi$k=1$ ) and $-0.3425>w\left(r_{h}\right)>-0.5027,0.9938<M<1.0052,0.5932>\delta_{o}>0.5831$ (cf. figs. 2c-d). For small $\mu$, the deviation from the Schwarzschild mass $M=0.5$ appears to scale with $\mu^{2}$ for the quasi- $k=0$ solutions; this can be understood from (3.39), which for $w \lesssim+1$ gives $m^{\prime}\left(r_{h}\right) \approx 4 \mu^{2}$ independent of $r_{h}$.

As we vary $r_{h} \rightarrow 0$, we find that the even- $k$ black hole solutions reduce to the regular solutions for the same value of $\mu$ in the manner of [18], with the shooting parameter approaching $w\left(r_{h}\right)=-1+b r_{h}^{2}$, where $b$ is the regular solution shooting parameter. The value of $\mu$ at which the two solution classes bifurcate also increases as $r_{h} \rightarrow 0$ from its value at $r_{h}=1$ to the regular solution bifurcation value; for $k=2,1.752 \times 10^{-2}<\mu_{b i f}<4.454 \times 10^{-2}$ as $0<r_{h}<1$. The reduction to the regular solutions is sensible when we examine eqs.(3.39)(3.41); despite the presence of the horizon, $w^{\prime}\left(r_{h}\right)$ and $\delta^{\prime}\left(r_{h}\right)$ approach the leading behavior of the $r \rightarrow 0$ regular solution expansions with $r$ replaced by $r_{h}$, while the non-horizon mass contribution $\left(2 m(r)-r_{h}\right) /\left(r-r_{h}\right)=4 b^{2} r_{h}^{2}$ mimics the regular solution mass-radius ratio $2 m(r) / r$ in the same limit.

Although for $\mu \neq 0$ there are no regular solution limits which the odd- $k$ black holes can approach as $r_{h} \rightarrow 0$, there is limiting behavior as the horizon continues to shield the $w(0)=$ +1 singularity. The shooting parameter for such solutions behaves as $w\left(r_{h}\right)=+1-b_{\text {eff }} r_{h}^{2}$, where $b_{\text {eff }}$ is now an effective regular shooting parameter, and we can determine bifurcation $\mu$ values as well as mass limits for the solution branches. In the $k=1$ case with $r_{h}=10^{-3}$, for example, we find for $0<\mu<0.2010$ the branches $0<b_{\text {eff }}<0.1329,0<M<0.9289$ (quasi- 
$k=0)$ and $0.4537>b_{\text {eff }}>0.1329,0.8286<M<0.9289$; the latter branch reduces to the $k=1$ solution of [19] while former approaches the Schwarzschild solution with $M$ decreasing proportional to $r_{h}$. Because the curvature at the horizon $R_{\mu}{ }^{\mu}=-8 \pi T_{\mu}{ }^{\mu}=\mu^{2}(1+w)^{2} / r_{h}^{2}$ diverges as $r_{h} \rightarrow 0$, the maximum value of $\mu$ will presumably decrease until it falls off faster than $\sim r_{h}$, which will bring the expansions (3.40)-(3.41) and the non-horizon mass contribution into the regular solution form (we have not investigated this in detail). Thus the only legitimate quasi-regular limiting black hole solutions for odd $k$ occur for $\mu \rightarrow 0$, but these are significant: the $r_{h} \rightarrow 0$ odd-node black holes help "complete" the regular spectrum, in that the regular and quasi-regular solutions to ENAP theory interpolate between the Schwarzschild vacuum and the gravitating Dirac monopole [17]. As mentioned above, we will find that the quasi- $k=0$ branch of solutions, which are trivial apart from a rapid transition between the minima of the non-abelian self-coupling $w=+1$ and $w=-1$, exist with or without event horizons in EYMH theory and correspond to gravitating $S U(2)$ sphalerons.

It should be noted that the divergent behavior of $w$ as $r \rightarrow \infty$ used to shoot both regular and black hole solutions is characteristic of integration toward a singular point. We would observe the same qualitative behavior as $r \rightarrow 0$ or $r_{h}$ if we had chosen to integrate inward from some large value of $r$ using (3.32)-(3.34) as initial data and $\{c, M\}$ as shooting parameters. To help verify the existence of our solutions, we integrated from both small $r$ and large $r$ to a point inbetween, where we attempted to match the values of $w, w^{\prime}$, and $m$ by adjusting the shooting parameters $\{b, c, M\}$. Using the value of $b$ found from our original method, we were able to adjust $\{c, M\}$ to give agreement at the common point to an accuracy comparable to the global tolerance. We repeated this procedure for EYMH solutions after including additional parameters associated with the Higgs field.

\section{EYMH Theory: Regular and Black Hole Solutions}

\section{The Higgs Ansatz}

The starting point for our theory is the addition in eq.(2.8) of a complex scalar Lagrangian to the ordinary YM Lagrangian. Following the standard model, we take the Higgs field to be a complex doublet $\Phi=\left(\phi^{+}, \phi^{-}\right)$with the double-well self-interaction

$$
V(\Phi)=\lambda\left(\Phi^{\dagger} \Phi\right)^{2}-\mu^{2}\left(\Phi^{\dagger} \Phi\right)+\text { constant }
$$


The most general complex doublet Higgs may be parameterized

$$
\Phi(x) \equiv \frac{1}{\sqrt{2}} \exp [-\tau \cdot \xi(x)]\left(\begin{array}{c}
0 \\
h(x) / r
\end{array}\right)
$$

with $h$ and $\xi^{a}$ arbitrary. A parameterization of the Higgs field which proves useful in finding spherically symmetric EYMH solutions can be achieved by rewriting $\Phi$ in the form

$$
\Phi(x)=\frac{1}{\sqrt{2}}\left(\begin{array}{c}
\psi_{2}(x)+i \psi_{1}(x) \\
\phi(x)-i \psi_{3}(x)
\end{array}\right),
$$

where we have grouped three of the degrees of freedom as a vector $\psi$. Substituting into the EYMH Lagrangian (2.8) gives

$$
\begin{aligned}
\mathcal{L}_{\mathrm{EYMH}}=-\frac{1}{4 \pi} & {\left[\frac{1}{4}|F|^{2}+\frac{1}{8}\left(\phi^{2}+|\psi|^{2}\right)|A|^{2}\right.} \\
& +\frac{1}{2} g^{\mu \nu}\left[\partial_{\mu} \phi \partial_{\nu} \phi+\left(\partial_{\mu} \psi\right) \cdot\left(\partial_{\nu} \psi\right)\right]+V\left(\phi^{2}+|\psi|^{2}\right) \\
& \left.+\frac{1}{2} g^{\mu \nu} A_{\mu} \cdot\left(\psi \times \partial_{\nu} \psi+\psi \partial_{\nu} \phi-\phi \partial_{\nu} \psi\right)\right],
\end{aligned}
$$

where $A$ is our original connection ansatz (3.7) . A useful ansatz for the Higgs field which yields a spherically symmetric energy density is to take $\phi=\phi(r, t)$ and $\psi=\psi(r, t) \widehat{n}_{r}$, with $\widehat{n}_{r}$ a unit vector in the radial direction.

The static field equations in the gauge $b \equiv 0$, without the ansatz $a \equiv d \equiv 0$, are

$$
\begin{aligned}
& \frac{d}{d r}\left[r^{2} \frac{T}{R} a^{\prime}\right]-2 R T\left[\left(w^{2}+d^{2}\right)+\frac{1}{8} r^{2}\left(\phi^{2}+\psi^{2}\right)\right] a=0 \\
& \frac{d}{d r}\left[\frac{w^{\prime}}{R T}\right]+\frac{\left[1-\left(w^{2}+d^{2}\right)\right]}{r^{2}} w \frac{R}{T}-\frac{1}{4}\left[(1+w) \phi^{2}-(1-w) \psi^{2}\right] \frac{R}{T}+a^{2} w R T=0 \\
& \frac{d}{d r}\left[\frac{d^{\prime}}{R T}\right]+\frac{\left[1-\left(w^{2}+d^{2}\right)\right]}{r^{2}} d \frac{R}{T}-\frac{1}{4}\left[\left(\phi^{2}+\psi^{2}\right) d-2 \phi \psi\right] \frac{R}{T}+a^{2} d R T=0 \\
& \frac{d}{d r}\left[\frac{r^{2} \phi^{\prime}}{R T}\right]-V_{\phi}^{\prime} r^{2} \frac{R}{T}-\frac{1}{2}\left(\left[(1+w)^{2}+d^{2}\right] \phi-2 d \psi\right) \frac{R}{T}+\frac{1}{4} a^{2} r^{2} \phi R T=0 \\
& \frac{d}{d r}\left[\frac{r^{2} \psi^{\prime}}{R T}\right]-V_{\psi}^{\prime} r^{2} \frac{R}{T}-\frac{1}{2}\left(\left[(1-w)^{2}+d^{2}\right] \psi-2 d \phi\right) \frac{R}{T}+\frac{1}{4} a^{2} r^{2} \psi R T=0,
\end{aligned}
$$


with the constraint equation

$$
\frac{2}{R T}\left[\left(d^{\prime} w-w^{\prime} d\right)+\frac{1}{2} r^{2}\left(\phi^{\prime} \psi-\psi^{\prime} \phi\right)\right]=0
$$

arising from the gauge choice. Following Witten [26], it is useful to express the $w$ and $d$ degrees of freedom in complex scalar form:

$$
w(r)-i d(r)=f(r) \exp i \alpha(r)
$$

Substitution of $w=f \cos \alpha$ and $d=-f \sin \alpha$ into the constraint equation with $\phi \equiv \psi \equiv 0$, $v \equiv 0$ gives the result $f^{2} \alpha^{\prime}=0$, implying that the gauge choice allows for the dynamical elimination of one of the remaining degrees of freedom: $w$ and $d$ are related by a multiplicative constant (the equations for $d$ and $w$ in EYM theory are identical), and $d \equiv 0$ in [19] and $[18]$ is not an ansatz but a result of the choice of the constant $\alpha \equiv 0[19,18]$. With $\phi$ and $\psi$ nonzero, the constraint equation becomes

$$
2 f^{2} \alpha^{\prime}+\frac{1}{4} h^{2} \gamma^{\prime}=0
$$

Examination of the field equations then implies that if either $\alpha$ or $\gamma$ is chosen to be a constant, then $\alpha-\gamma=n \pi$.

We will only study the case in which $\alpha$ and $\gamma$ are constants, and hence obtaining static solutions to EYMH theory reduces to solving the coupled equations for $a, f$, and $h / r$ and choosing $\alpha$ and $n$.

The equation for a may be rewritten

$$
\frac{1}{2} \frac{d}{d r}\left[r^{2} \frac{T}{R}\left(a^{2}\right)^{\prime}\right]=r^{2} \frac{T}{R}\left(a^{\prime}\right)^{2}+2 R T\left[f^{2}+\frac{1}{8} h^{2}\right] a^{2}
$$

while the $f$ and $h / r$ equations become

$$
\begin{aligned}
& \frac{d}{d r}\left[\frac{f^{\prime}}{R T}\right]+\frac{\left[1-f^{2}\right] f}{r^{2}} \frac{R}{T}-\frac{1}{4}\left(\frac{h}{r}\right)^{2}[f+\cos n \pi] \frac{R}{T}+a^{2} f R T=0 \\
& \frac{d}{d r}\left[\frac{r^{2}(h / r)^{\prime}}{R T}\right]-V_{h}^{\prime} r^{2} \frac{R}{T}-\frac{1}{2}[f+\cos n \pi]^{2}\left(\frac{h}{r}\right) \frac{R}{T}+\frac{1}{4} a^{2} r^{2}\left(\frac{h}{r}\right) R T=0 .
\end{aligned}
$$

For odd $n$, the above equations can be put in the same form as the even- $n$ equations by defining $\tilde{f} \equiv-f$, so the solutions for odd and even $n$ are isomorphic despite the fact that 
the physical field configurations are quite different. Before exploring these differences, we note that the $r \rightarrow \infty$ boundary conditions on $f$ and $h$ require $a(\infty)=0$, and for regular solutions $a(0)=0$. Since the right side of (4.13) is positive semi-definite, we must have $a \equiv 0$ for static regular solutions. Thus the no-dyon results for EYM theory [32] extend to EYMH theory.

Though solutions to (4.14)-(4.15) do not depend explicitly on the values of $\alpha$ and $n$, some physical properties of the Higgs field and connection are affected by these parameters. Consider $\alpha=0$, for example. The even- $n$ solutions reduce to our ansatz, while the odd- $n$ solutions have have a "hedgehog" Higgs field $(\phi, \psi) \equiv(0, \pm h / r)$. We will shortly see that one of the smooth $k=1$ node solutions of this form which we find in the next section has a limit in which it becomes close to the flat space sphaleron of YMH theory.

\section{EYMH Equations and Boundary Conditions}

To obtain static, spherically symmetric solutions we assume $\phi=\phi(r)=v+\eta(r)$ and $\psi=0$. The Lagrangian for the theory may then be written in the form

$$
\mathcal{L}_{\mathrm{EYMH}}=-\frac{1}{4 \pi}\left(\frac{1}{4}|F|^{2}+\frac{1}{2}\left(\frac{g \phi}{2}\right)^{2}|A|^{2}+\frac{1}{2}|\partial \eta|^{2}+V(\phi)\right)
$$

where $V(\phi)=\lambda\left[\phi^{2}-v^{2}\right]^{2} / 4$ satisfies $V( \pm v)=0$.

Specializing our results of the last section, the gauge field equation is

$$
\frac{d}{d r}\left(\frac{w^{\prime}}{R T}\right)+\frac{w\left(1-w^{2}\right)}{r^{2}} \frac{R}{T}-\left(\frac{g \phi}{2}\right)^{2}(1+w) \frac{R}{T}=0
$$

and the Higgs equation is

$$
\frac{d}{d r}\left(\frac{r^{2} \eta^{\prime}}{R T}\right)-\frac{1}{2}(1+w)^{2} \phi \frac{R}{T}-V^{\prime}(\phi) r^{2} \frac{R}{T}=0
$$

where $V^{\prime} \equiv d V / d \phi=d V / d \eta$.

From variation of the full action with respect to $g_{\mu \nu}$ we obtain the energy-momentum 
tensor,

$$
\begin{aligned}
8 \pi T_{\mu \nu}=2 F_{\mu \gamma} F_{\nu}^{\gamma} & -\frac{1}{2} g_{\mu \nu}|F|^{2}+\left[2\left(\frac{g \phi}{2}\right)^{2} A_{\mu} A_{\nu}-g_{\mu \nu}\left(\frac{g \phi}{2}\right)^{2}|A|^{2}\right] \\
& +2 \partial_{\mu} \eta \partial_{\nu} \eta-g_{\mu \nu}\left[|\partial \eta|^{2}+2 V(\phi)\right]
\end{aligned}
$$

and the $(t)$ and $(r r)$ Einstein equations:

$$
\begin{aligned}
m^{\prime}= & \left(1-\frac{2 m}{r}\right)\left(\frac{w^{\prime}}{g}\right)^{2}+\frac{1}{2} \frac{\left(1-w^{2}\right)^{2}}{g^{2} r^{2}}+\left(\frac{\phi}{2}\right)^{2}(1+w)^{2} \\
& +\frac{1}{2}\left(1-\frac{2 m}{r}\right)\left(r \eta^{\prime}\right)^{2}+V(\phi) r^{2} \\
r\left(1-\frac{2 m}{r}\right) \frac{T^{\prime}}{T}= & -\left(1-\frac{2 m}{r}\right)\left(\frac{w^{\prime}}{g}\right)^{2}+\frac{1}{2} \frac{\left(1-w^{2}\right)^{2}}{g^{2} r^{2}}+\left(\frac{\phi}{2}\right)^{2}(1+w)^{2} \\
& -\frac{1}{2}\left(1-\frac{2 m}{r}\right)\left(r \eta^{\prime}\right)^{2}+V(\phi) r^{2}-\frac{m}{r} .
\end{aligned}
$$

For black hole solutions, we again replace the auxiliary $T^{\prime}$ equation with an equation for $\delta^{\prime}$,

$$
\delta^{\prime}=-\left[2\left(w^{\prime} / g\right)^{2}+\left(r \eta^{\prime}\right)^{2}\right] / r
$$

The Einstein equations can once again be used to express the equations of motion in a form independent of $g_{t t}$ :

$$
\begin{aligned}
r^{2}\left(1-\frac{2 m}{r}\right) w^{\prime \prime}+ & {\left[2 m-\frac{\left(1-w^{2}\right)^{2}}{g^{2} r}-2\left(\frac{\phi}{2}\right)^{2}(1+w)^{2} r-2 V(\phi) r^{3}\right] w^{\prime} } \\
+ & \left(1-w^{2}\right) w-\left(\frac{g \phi}{2}\right)^{2}(1+w) r^{2}=0 \\
r^{2}\left(1-\frac{2 m}{r}\right) \eta^{\prime \prime}+ & {\left[2(r-m)-\frac{\left(1-w^{2}\right)^{2}}{g^{2} r}-2\left(\frac{\phi}{2}\right)^{2}(1+w)^{2} r-2 V(\phi) r^{3}\right] \eta^{\prime} } \\
- & \frac{1}{2}(1+w)^{2} \phi-V^{\prime}(\phi) r^{2}=0 .
\end{aligned}
$$

The addition of the Higgs field and new coupling constants $\lambda, \mu^{2}$ requires a reexamination of the scaling properties of the field equations. From the replacement of $\mu^{2}$ in the ENAP 
theory with $(g \phi / 2)^{2}$ and examination of $\mathcal{L}_{\mathrm{EYMH}}$, we find

$$
[\eta]=[v]=1, \quad[\lambda]^{1 / 2}=[\mu]=[L]^{-1},
$$

so by introducing $\widehat{\lambda}=\lambda / g^{2}$ and $\widehat{\mu}=\mu / g$, we obtain the same overall scaling behavior of the Lagrangian found in section III:

$$
\mathcal{L}_{\mathrm{EYMH}}=g^{2} \mathcal{L}_{\mathrm{EYMH}}(\widehat{r}, \widehat{\lambda}, \widehat{\mu})
$$

Solutions for $g \neq 1$ will again be related to dimensionless quantities as before, with the addition of $\eta_{g}=\eta(g r)$, and the dimensionless field equations can be obtained by replacing $(r, m, \lambda, \mu)$ with $(\widehat{r}, \widehat{m}, \widehat{\lambda}, \widehat{\mu})$ and setting $g=1$ in eqs.(4.20)-(4.24). We take $g=1$ for the remainder of the paper and consider all quantities dimensionless unless otherwise specified.

By following the analysis of section III, we can again predict general features of the solutions and boundary conditions. From the expression for $\delta(4.22)$, we see that $R / T$ again increases (and $\delta$ for black holes decreases) monotonically with radius, but because $T$ now satisfies

$$
\frac{d}{d r}\left[\frac{r^{2}}{R}\left(\frac{1}{T}\right)^{\prime}\right]=2\left(1-\frac{2 m}{r}\right)\left(w^{\prime}\right)^{2} \frac{R}{T}+\left(\frac{R}{r^{2} T}\right)\left[\left(1-w^{2}\right)^{2}-2 V(\phi)\right]
$$

it is no longer clear whether $T$ decreases monotonically for regular solutions. The condition $T>R \geq 1$, with $R$ possessing at least one maximum, still holds for regular solutions.

The YM equation may be rewritten

$$
\frac{1}{2} \frac{d}{d r}\left[\frac{\left(w^{2}\right)^{\prime}}{R T}\right]=\frac{\left(w^{\prime}\right)^{2}}{R T}+\frac{R}{r^{2} T}\left[\left(w^{2}-1\right) w^{2}+\left(\frac{\phi}{2}\right)^{2}(1+w) w r^{2}\right],
$$

in general, while at turning points we have the relation

$$
w w^{\prime \prime}=\frac{R^{2}}{r^{2}}(1+w) w\left[w(w-1)+\left(\frac{\phi}{2}\right)^{2} r^{2}\right] \quad\left(w^{\prime}=0\right)
$$

Since $\phi(\infty) \neq 0$ for a spontaneously broken theory, $w=-1$ is again the only acceptable asymptotic value and $w^{2}<1$ is required for finite energy solutions. Because $\phi$ is a field 
and not a constant, determining restrictions on the value of $w$ and the occurrence of turning points is not as simple as for ENAP theory. If we assume that $\phi$ is $\mathcal{O}(v)$ in the region $r \gtrsim 1$, we can again use a characteristic radius to define two regimes of interest and apply the analysis of section III:

$$
\begin{array}{ll}
r \lesssim \mathcal{O}(1 / v): & -1<w<\frac{1}{2}\left(1+\sqrt{1-(v r)^{2}}\right) \\
r \gtrsim \mathcal{O}(1 / v): & w^{\prime}<0, \quad w^{\prime \prime}>0
\end{array}
$$

quantify the finite-energy constraints on both regular and black hole solutions.

To better understand the expected behavior of $\phi$, we rewrite the Higgs equation

$$
\frac{1}{2} \frac{d}{d r}\left[\frac{r^{2}\left(\phi^{2}\right)^{\prime}}{R T}\right]=\frac{\left(r \phi^{\prime}\right)^{2}}{R T}+\frac{R}{T} r^{2}\left[\frac{(1+w)^{2}}{2 r^{2}} \phi^{2}+V^{\prime}(\phi) \phi\right] .
$$

The obvious requirement for finite energy solutions is $V^{\prime}(\phi) \phi \leq 0$, which implies that $\phi$ is restricted to lie between the minima of the potential: $|\phi| \leq v$. We also note that $\phi= \pm v, 0$ are the only allowed values as $r \rightarrow \infty$, but because $V(\phi)$ is nonzero at $\phi(\infty)=0$, finite energy solutions must have $\phi(\infty)= \pm v$. From (4.31), the equation governing the oscillatory properties of $\phi$ is

$$
\phi \phi^{\prime \prime}=R^{2}\left[\frac{(1+w)^{2}}{2 r^{2}} \phi^{2}+V^{\prime}(\phi) \phi\right] \quad\left(\phi^{\prime}=0\right) .
$$

When the gauge field coupling term is negligible, we see that $\phi \phi^{\prime \prime}<0$, which is characteristic of oscillations about $\phi=0$ (a solution of infinite energy) unless the initial value of $\phi^{\prime}$ in (4.31) is large enough for the field to reach one of the minima of $V(\phi)$ as $\phi^{\prime} \rightarrow 0$. For regular solutions, we will find that $\phi^{\prime}(0)=0$ for $\phi(0) \neq 0$, so oscillatory behavior will occur unless the gauge field coupling becomes important. For black hole solutions and regular solutions with $\phi(0)=0$, the initial derivative of $\phi$ is nonzero, but the gauge field term again plays an important role in avoiding infinite energy solutions. The behavior of the scalar field is consistent with the analogue of a particle moving in a potential proportional to $-V$, with the gauge field interaction and gravitational forces fighting the restoring force of the potential. These heuristic considerations are in agreement with the no-hair proof for the Goldstone theory [2], which approaches the existence of finite energy solutions rigorously 
but for which the analysis fails in the presence of a non-abelian gauge field. Thus we expect the Higgs field either to smoothly transit the potential until $|\phi|=v$, or to move initially toward $\phi=0$ and then turn and roll under the influence of the gauge field toward $|\phi|=v$. The $\phi \rightarrow-\phi$ symmetry of the field equations also implies that every solution will have a mirror-image solution; we focus here on solutions with $\phi(\infty)=+v$ without loss of generality.

The presence of the Higgs field in EYMH theory allows for two possible sets of initial conditions for regular solutions. Finite $T_{t t}$ and regularity of the metric at $r=0$ give

$$
\begin{aligned}
2 m(r) & =\mathcal{O}\left(r^{3}\right) \\
\ln T(r) & =\mathcal{O}\left(r^{2}\right) \\
\left(\begin{array}{l}
w(r) \\
\eta(r)
\end{array}\right) & =\left(\begin{array}{c}
-1+\mathcal{O}\left(r^{2}\right) \\
\eta_{o}+\mathcal{O}\left(r^{2}\right)
\end{array}\right) \quad \text { Even }-\mathrm{k} \\
\left(\begin{array}{l}
w(r) \\
\eta(r)
\end{array}\right) & =\left(\begin{array}{c}
+1+\mathcal{O}\left(r^{2}\right) \\
-v+\mathcal{O}(r)
\end{array}\right) \quad \text { Odd }-\mathrm{k},
\end{aligned}
$$

where $k$ again denotes the number of $w$ nodes and $-v<\eta_{o}<0$ for $\phi(\infty)=+v$. Black hole solutions again possess expansions like eqs.(3.29) - (3.31) near the horizon, with the addition of

$$
\eta(r)=\eta\left(r_{h}\right)+\eta^{\prime}\left(r_{h}\right)\left(r-r_{h}\right)+\mathcal{O}\left(r-r_{h}\right)^{2}
$$

where $\eta\left(r_{h}\right)$ brings to three the number of unknowns.

The vacuum values $w(\infty)=-1$ and $\eta(\infty)=0$ are shared by black hole and regular solutions; the behavior of the field equations as $r \rightarrow \infty$ gives

$$
\begin{aligned}
m(r) & \sim M-\frac{1}{2} a^{2}(\sqrt{2} \mu r) r e^{-2 \sqrt{2} \mu r} \\
\ln T(r) & \sim \ln \left(\frac{1}{T_{o}}\right)+\frac{M}{r} \\
\delta(r) & \sim-\delta_{o}+\frac{1}{2} a^{2}(\sqrt{2} \mu r) e^{-2 \sqrt{2} \mu r} \\
w(r) & \sim-1+c e^{-(v / 2) r} \\
\eta(r) & \sim a e^{-\sqrt{2} \mu r}
\end{aligned}
$$


where $c$ and $a$ are constants, $v / 2$ is the gauge field mass after symmetry breaking and $\sqrt{2} \mu$ is the Higgs field mass.

\section{Numerical Regular Solutions}

The presence of the Higgs field makes the solution of the EYMH equations a twoparameter shooting problem. For even- $k$ solutions, the boundary conditions (4.33)-(4.35) become

$$
\begin{aligned}
2 m(r)= & \left(4 b^{2}+\frac{2}{3} V_{o}\right) r^{3}+ \\
& \frac{2}{5}\left[-8 b^{3}+\left(3\left(\frac{\phi_{o}}{2}\right)^{2}+\frac{16}{3} V_{o}\right) b^{2}+8\left(\frac{1}{6} V_{o}^{\prime}\right)^{2}\right] r^{5}+\mathcal{O}\left(r^{7}\right) \\
\ln T(r)= & \left(2 b^{2}-\frac{1}{3} V_{o}\right) r^{2} \\
& -\left(\frac{1}{5}\left[12 b^{4}-4 b^{3}+\left(\left(\frac{\phi_{o}}{2}\right)^{2}+4 V_{o}\right) b^{2}-3\left(\frac{1}{6} V_{o}^{\prime}\right)^{2}\right]-\frac{1}{9} V_{o}^{2}\right) r^{4}+\mathcal{O}\left(r^{6}\right) \\
w(r)= & \left.-1+b r^{2}+\frac{1}{10}\left[8 b^{3}-3 b^{2}+\left(\frac{\phi_{o}}{2}\right)^{2}+4 V_{o}\right) b\right] r^{4}+\mathcal{O}\left(r^{6}\right) \\
\eta(r)= & \eta_{o}+\frac{1}{6} V_{o}^{\prime} r^{2}+\left[\frac{1}{120} V_{o}^{\prime}\left(24 b^{2}+\frac{20}{3} V_{o}+V_{o}^{\prime \prime}\right)+\frac{1}{40} \phi_{o} b^{2}\right] r^{4}+\mathcal{O}\left(r^{6}\right)
\end{aligned}
$$

where $V_{o}, V_{o}^{\prime}$ and $V_{o}^{\prime \prime}$ are the potential and its derivatives with respect to $\phi$ at $\phi=\phi_{o} \equiv v+\eta_{o}$, and the shooting parameters are $b>0$ and $\eta_{o}$. For odd- $k$ solutions, the expansions are

$$
\begin{aligned}
2 m(r)= & \left(4 b^{2}+\frac{2}{3} V_{o}+\left(\eta_{o}^{\prime}\right)^{2}\right) r^{3}+ \\
& \frac{2}{5}\left(-8 b^{3}+\frac{16}{3} V_{o} b^{2}+\left(\eta_{o}^{\prime}\right)^{2}\left[6 b^{2}-3 b+\left(\left(\eta_{o}^{\prime}\right)^{2}+V_{o}+V_{o}^{\prime \prime}\right)\right]\right) r^{5}+\mathcal{O}\left(r^{7}\right) \\
\ln T(r)= & \left(2 b^{2}-\frac{1}{3} V_{o}\right) r^{2}+\frac{1}{9} V_{o}^{2} r^{4} \\
& -\frac{1}{5}\left(12 b^{4}-4 b^{3}+4 V_{o} b^{2}+\frac{1}{4}\left(\eta_{o}^{\prime}\right)^{2}\left[24 b^{2}-2 b-\frac{8}{3} V_{o}-V_{o}^{\prime \prime}\right]\right) r^{4}+\mathcal{O}\left(r^{6}\right) \\
w(r)= & +1-b r^{2}-\frac{1}{10}\left(8 b^{3}-3 b^{2}+4 V_{o} b-\frac{1}{2}\left(\eta_{o}^{\prime}\right)^{2}[1-8 b]\right) r^{4}+\mathcal{O}\left(r^{6}\right) \\
\eta(r)= & \eta_{o}^{\prime} r+\frac{1}{10} \eta_{o}^{\prime}\left(8 b^{2}-2 b+3\left(\eta_{o}^{\prime}\right)^{2}++\frac{8}{3} V_{o}+V_{o}^{\prime \prime}\right) r^{3}+\mathcal{O}\left(r^{5}\right)
\end{aligned}
$$

where $\eta_{o}^{\prime}$ replaces $\eta_{o}$ as the second shooting parameter. We again evaluate the initial conditions at $r=10^{-3}$ and use global error tolerance $10^{-12}$ in a standard ordinary differential 
equation solver, adjusting either $\left(b, \eta_{o}\right)$ or $\left(b, \eta_{o}^{\prime}\right)$ for fixed $\lambda$ and $\mu$ and integrating toward $r=\infty$. The finite energy solution bracketing condition for $b$ is similar to that of ENAP theory: for $\eta_{o}$ or $\eta_{o}^{\prime}$ in the vicinity of a solution, there exists a range of $b$ for which $w \rightarrow-1$ but then rapidly approaches $|w|=\infty$, with a discrete value of $b$ giving the correct asymptotics for $w$. As we adjust $\eta_{o}$ or $\eta_{o}^{\prime}$, the Higgs field either passes through $\eta=0$ and diverges or experiences a turning point and begins oscillating about $\eta=-v$, with discrete values of $\left(b, \eta_{o}\right)$ or $\left(b, \eta_{o}^{\prime}\right)$ giving the monotonic approach of both fields toward the boundary values as $r \rightarrow \infty$. The difficulty of the two-dimensional shooting problem was compounded by the presence of the two free parameters $\lambda$ and $\mu$. Examination of (4.41)-(4.42)reveals that a significant disparity between $\sqrt{2} \mu$ and $v / 2$ makes the accurate determination of solution shooting parameters very difficult, since both fields must simultaneously approach their asymptotic values for both bracketing conditions to occur. As a consequence, we focus on solutions with $\sqrt{2} \mu=v / 2$ (corresponding to $\lambda=1 / 8$ ).

The general properties of finite energy regular solutions are the same as for ENAP theory. Solutions are again characterized by $w$ oscillations in the region $r \gtrsim 1$ and classified by the node number $k$, which may now be odd as well as even. For odd $k$, the Higgs deviation monotonically increases in the range $-v \leq \eta \leq 0$, while for even $k$ it undergoes a turning point very close to $\eta_{o}$ and then monotonically increases to $\eta(\infty)=0$. Two distinct sheets of solutions for each $k$ again arise (for any choice of $\lambda$ or $\mu$ in the appropriate range we have two solutions for each choice of $k$ ), but for $\lambda=1 / 8$ they do not precisely converge, and a different maximum value $v_{\max }$ for each sheet may be determined. Though we have not fully investigated this phenomenon, these two sheets corresponding to the different solution classes presumably bifurcate at a point with $\lambda_{b i f} \neq 1 / 8$. The results for $k=1$ and $k=2$ are shown in fig. $3 a-d$. As $v$ varies in the range $0<v<0.599$ for the first $k=1$ branch, the shooting parameters vary over $0.454>b>0.261$ and $0.323 v<\eta_{o}^{\prime}<0.329 v_{\max }$ and the mass behaves as $0.8286<M<0.9272$; the $v \rightarrow 0$ limit is the $k=1$ solution of [19]. The quasi$k=0$ branch is described by $0<v<0.619,0.292 v^{2}<b<0.206,0.434 v^{2}<\eta_{o}^{\prime}<0.307 v_{\max }$, and $1.821 v<M<0.9380$. This approaches the weak-gravity regime quickly as $v$ decreases: $g_{t t}(0)$ increases from its minimum value 0.1557 at $v_{\max }$ to $g_{t t}(0)=0.9998$ at $v=10^{-2}$. We also note that the scaling of the mass with $v$ is characteristic of sphaleron solutions to YMH theory, but address the relationship between our solutions and sphalerons more fully below. For $k=2$, the two solution branches are described by $0<v<0.120,0.652>b>0.585$, 
$-.870 v<\eta_{o}<-.845 v_{\max }, 0.9713<M<0.9972$ and $0<v<0.122,0.454<b<0.568$, $-v<\eta_{o}<-.850 v_{\max }, 0.8286<M<0.9982$ (quasi- $k=1$ ). As $v \rightarrow 0$, the branches approach the $k=2$ and $k=1$ solutions of [19], with the initial Higgs deviation of the quasi- $k=1$ branch approaching the odd- $k$ initial value $\eta_{o}=-v$.

We can again understand in heuristic terms the bifurcation of the solution space into two branches by appealing to length scales [33]. The coupling parameters in this model provide us with two length scales: $L_{1}=g^{-1} G^{1 / 2}$ and $L_{2}=g^{-1} \sqrt{\lambda / \mu^{2}}$ (where we do not set $\mathrm{G}=1$ for the remainder of this paragraph). As in our discussion of ENAP theory, the former of these scales is set by gravity while the latter is not. One branch of our solutions has its scale (the position after which the fields rapidly approach their asymptotic values) set by $L_{1}$ and the latter by $L_{2}$. In the limit that the $v$ goes to zero the k node solutions on the latter branch have their last node pushed out to infinity and they go over to $k-1$ node solutions with scale set by $L_{1}$. An interesting special case is that of our $k=1$ node solutions (smooth or black hole) since they would appear to approach a $k=0$ node solution. This means that their scale is set totally by $L_{2}$ and hence can exist with arbitrarily weak gravity. In particular, our smooth solution of this sort can be related to the flat space sphaleron in this limit. Explicitly, taking our solution with the choice $\alpha=0$ and odd $-n$ we can write the solution in the form

$$
\begin{aligned}
A_{\mu} & =\frac{(1+f)}{2} U \partial_{\mu} U^{-1} \\
\Phi & =\frac{1}{\sqrt{2}}\left(\frac{h}{r}\right) U\left[\begin{array}{l}
0 \\
1
\end{array}\right] \\
U & =\exp \left[-n \pi \widehat{\tau}_{r}\right],
\end{aligned}
$$

which is physically equivalent to the YMH sphaleron ansatz of [34] and [35]. Though the sphaleron solutions utilizing (4.51) have only been found for $k=1$ in YMH theory, we see that they appear to exist for all $k$ when we include gravity.

\section{Numerical Black Hole Solutions}

To find numerical black hole solutions, we used the conditions

$$
m^{\prime}\left(r_{h}\right)=\left[1-w^{2}\left(r_{h}\right)\right]^{2} / 2 r_{h}^{2}+\left[\frac{\phi\left(r_{h}\right)}{2}\right]^{2}\left[1+w\left(r_{h}\right)\right]^{2}+V\left(\phi\left(r_{h}\right)\right) r_{h}^{2}
$$




$$
\begin{aligned}
w^{\prime}\left(r_{h}\right) & =\frac{\left[\phi\left(r_{h}\right) / 2\right]^{2}\left[1+w\left(r_{h}\right)\right] r_{h}^{2}-\left[1-w^{2}\left(r_{h}\right)\right] w\left(r_{h}\right)}{r_{h}-\left[1-w^{2}\left(r_{h}\right)\right]^{2} / r_{h}-2\left[\phi\left(r_{h}\right) / 2\right]^{2}\left[1+w\left(r_{h}\right)\right]^{2} r_{h}-2 V\left(\phi\left(r_{h}\right)\right) r_{h}^{3}} \\
\eta^{\prime}\left(r_{h}\right) & =\frac{\left[1+w\left(r_{h}\right)\right]\left[\phi\left(r_{h}\right) / 2\right]+V^{\prime}\left(\phi\left(r_{h}\right)\right) r_{h}^{2}}{r_{h}-\left[1-w^{2}\left(r_{h}\right)\right]^{2} / r_{h}-2\left[\phi\left(r_{h}\right) / 2\right]^{2}\left[1+w\left(r_{h}\right)\right]^{2} r_{h}-2 V\left(\phi\left(r_{h}\right)\right) r_{h}^{3}} \\
\delta^{\prime}\left(r_{h}\right) & =-\left(2\left[w^{\prime}\left(r_{h}\right)\right]^{2}+\left[r_{h} \eta^{\prime}\left(r_{h}\right)\right]^{2}\right) / r_{h}
\end{aligned}
$$

on the horizon, and use $w\left(r_{h}\right)$ and $\eta\left(r_{h}\right)$ as shooting parameters for $r_{h}=1$. The results for $k=1$ and $k=2$ are shown in figs. $4 a-d$.

Two solution branches again appear for each $k$, with the quasi- $k=0$ branch distinguished by its weak-gravity $v \rightarrow 0$ limit. The $k=1$ solutions are decribed by $0<v<0.356$, ( $1-$ $\left.0.292 v^{2}\right)>w\left(r_{h}\right)>0.870, \quad-(1-0.217 v) v<\eta\left(r_{h}\right)<-0.304, \quad(0.5+1.820 v)<M<$ 1.0181, $1.19 v^{2}<\delta_{o}<0.3310$ (quasi- $k=0$ ) and $0<v<0.331, \quad 0.632<w\left(r_{h}\right)<$ $0.801, \quad-0.826 v>\eta\left(r_{h}\right)>-0.275, \quad 0.9372<M<1.0043,0.5485>\delta_{o}>0.4300$. The specifications of the $k=2$ solutions are $0<v<0.0486,-0.632<w\left(r_{h}\right)<-0.518$, $-(1-1.62 v) v>\eta\left(r_{h}\right)>-0.0429, \quad 0.9372<M<1.0073, \quad 0.5485<\delta_{o}<0.5799$ (quasi$k=1)$ and $0<v<0.0475, \quad-0.345>w\left(r_{h}\right)>-0.489, \quad-0.901 v>\eta\left(r_{h}\right)>-0.0417$, $0.9938<M<1.0066,0.5932>\delta_{o}>0.5844$.

We see that these black hole solutions have nontrivial gauge and Higgs field structure outside of the horizon. This could not happen if the gauge group were abelian - it relies, as discussed in section II, on the non-abelian nature of the field theory being studied here.

\section{Conclusions}

In this paper we have studied spherically symmetric classical solutions to $S U(2)$ nonabelian Proca theory and spontaneously broken gauge theory. Our main intent has been to exploit a gap in the known no-hair results - they do not necessarily apply to nonlinear field theories - to find black hole solutions, which have nontrivial structure outside of the horizon, in the most familiar and relevant kinds of field theories . In particular, we have presented strong numerical evidence indicating that such field theories, when coupled to Einstein gravity, do admit spherically symmetric black hole solutions in which the gauge and Higgs fields decay to their asymptotic values exponentially far from the hole, in contrast to previous expectations. 
An important physical question is whether these solutions are stable (although we reemphasize that the no-hair proofs have to do with existence of classical solutions, not with their stability). We have not exhaustively studied this question, but it does seem somewhat unlikely that they are stable. Our EYMH solutions have a fairly direct relation to sphaleron solutions (even though many of them involve strong gravity) and hence are probably unstable. In fact, the arguments of [27-21] and [36] as applied to the smooth and black hole solutions of EYM solutions [19,18] seem likely ensure that our solutions are unstable. At this time we have only definitively studied this question for the smooth lowest node ENAP solutions using linear stability analysis along the lines of [27] which does in fact show these solutions to be unstable. It would be of interest to carry out this analysis fully, especially in light of the natural suggestion [21] that a no-hair theorem might hold if one demands stability. It is also important to develop methods to deal with the latter possibility in a general setting.

Another interesting question is to try to understand at a more fundamental level why these smooth and black hole solutions exist. Recently, the authors of [37] have given some very interesting plausibility arguments to argue for the existence of the solutions of [19] and [18]. These arguments rely on properties of the Yang-Mills configuration space associated with the existence of large gauge transformations. However, one can evade the no-hair arguments in as simple a theory as two scalar fields so long as the quartic potential is chosen judiciously. It would be interesting to see if smooth and black hole solutions can be found in such models as this will help to clarify the essential physics. We are presently studying this question and will report on it elsewhere.

Acknowledgements: We would like to thank P. Argyres, T. Imbo, M. Ortiz, A. Shapere, D. Spector, H. Tye and S.T. Yau for helpful discussions. This work was supported in part by the National Science Foundation, and by DOE grant DE-AC02-76ER 03069. 


\section{REFERENCES}

1. J.D. Bekenstein, Phys.Rev. D 5(1972), 1239.

2. S.L. Adler and R.D. Pearson, Phys.Rev. D 18(1978), 2798.

3. C. Teitelboim, Phys.Rev. D 5(1972), 2941.

4. R.H. Price, Phys.Rev. D 5(1972), 2419.

5. S. Coleman, J. Preskill, and F. Wilczek, Nucl.Phys. B 378(1992), 175.

6. In this paper our concern is with classical "hair" only. We do not address the interesting but separate issue of quantum hair [5].

7. S. Coleman, J. Preskill, and F. Wilczek, Phys.Rev.Lett. 67(1991), 1975

8. G.W. Gibbons, "Self-gravitating Magnetic Monopoles, Global Monopoles and Black Holes", Lisbon 1990, Proceedings, The Physical Universe (1990)110.

9. References to sphaleron solutions in this paper denote the standard SU(2) YM solution with a Higgs doublet (rather than more general saddle points).

10. G.W. Gibbons and K. Maeda, Nucl.Phys. B 298(1988), 741.

11. D. Garfinkle, G.T. Horowitz and A. Strominger, Phys.Rev. D 43(1991), 3140.

12. J. Preskill, P. Schwarz, A. Shapere, S. Trivedi, F. Wilczek, Mod.Phys.Lett. A 6(1991), 2353.

13. A. Shapere, S. Trivedi and F. Wilczek, Mod.Phys.Lett. A 6(1991), 2677.

14. C. Holzey and F. Wilczek, Nucl.Phys B 380(1992), 447.

15. J.H. Horne and G.T. Horowitz, Phys.Rev. D 46(1992), 1340.

16. K. Lee, V.P. Nair, and E.J. Weinberg, Phys.Rev. D 45(1992), 2751.

17. M. Ortiz, Phys.Rev. D 45(1992), 2586.

18. P. Bizon, Phys.Rev.Lett. 64(1990), 2844.

19. R. Bartnik and J. McKinnon, Phys.Rev.Lett. 61(1988), 141.

20. S. Droz, M. Heusler, and N. Straumann, Phys.Lett. B 268(1991), 371.

21. Z. Zhou and N. Straumann, Nucl.Phys. B 369(1991), 180. 
22. J. Smoller, A. Wasserman, S.-T. Yau, and J. McLeod, Commun.Math.Phys. 143(1991), 115.

23. In [19] strong numerical evidence was given for the existence of such solutions. Subsequently, the existence of these solutions was rigorously established in [22].

24. S. Coleman, Commun.Math.Phys. 55(1977), 113.

25. S. Deser, Class.Quantum Grav. 1(1984), L1.

26. E. Witten, Phys.Rev.Lett. 38(1977), 121.

27. N. Straumann and Z. Zhou, Phys.Lett. B 237(1990), 353.

28. G. t'Hooft, Nucl.Phys. B 35(1971), 167.

29. We shall use the prefix "quasi-" to describe solutions when their $\mu \rightarrow 0$ behavior suggests a correspondence to some other solution category.

30. P. Bizon and T.Chmaj, "Gravitating Skyrmions", Wien U. preprint UWThPh-1992-23 (1992).

31. We recently became aware that a similar bifurcation of solutions has been observed in the Einstein-Skyrme (ES) system [30].

32. A.A. Ershov and D.V. Gal'tsov, Phys.Lett. A 150(1990), 159.

33. We note that in EYMH theory, we have only examined the one- and two-node solutions and hence it is possible that our discussion is sensitive to special features of these limited cases.

34. R.F. Dashen, B. Hasslacher, and A. Neveu, Phys.Rev. D 10(1974), 4138.

35. F.R. Klinkhamer and N.S. Manton, Phys.Rev. D 30(1984), 2212.

36. D.V. Gal'tsov and M.S. Volkov, Phys.Lett. A 162(1992), 144.

37. D. Sudarsky and R. Wald, "Extrema of Mass, Stationarity and Staticity, and Solutions to the Einstein-Yang-Mills Equations", U. of Chicago preprint (1992). 


\section{FIGURE CAPTIONS}

1) Two-node regular solutions to Einstein-Non-Abelian-Proca theory. The connection $(1+w(r))$ and total mass are plotted as functions of radius for a range of vector field mass values $0<\mu<\mu_{\max }$. Increasing $\mu$ in figures (1a) and (1b) (and in figure (2)) corresponds to decreasing the value of the radius at which the solution exponentially decays to its vacuum value. The $k=2$ and quasi- $k=1$ solutions, so named because as $\mu \rightarrow 0$ they resemble the $k=1$ solution of Bartnik and McKinnon [19], bifurcate at $\mu=\mu_{\max }$

2) One- and two-node black hole solutions to Einstein-Non-Abelian-Proca theory for horizon radius $r_{h}=1$. Like the regular case, both odd and even-node solutions exhibit bifurcation at a maximum value of $\mu$. The quasi- $k=0$ solutions resemble the Schwarzschild solution $w=1$ as $\mu \rightarrow 0$; this is the only class of ENAP solutions with a weak-gravity limit.

3) One- and two-node regular solutions to Einstein-Yang-Mills-Higgs theory for quartic coupling $\lambda=1 / 8$ and a range of vacuum expectation values $v$. Here and in figure (4), increasing $v$ corresponds to decreasing the value of the radius at which the solution exponentially decays to its vacuum value. As $v$ decreases from its maximal value, the quasi- $k=0$ solutions approach a flat space limit which is the well known Yang-Mills sphaleron. The other solutions do not admit a weak gravity limit.

4) One- and two-node black hole solutions to Einstein-Yang-Mills-Higgs theory for quartic coupling $\lambda=1 / 8$, a range of vacuum expectation values $v$ and $r_{h}=1$. The quasi$k=0$ solution approaches a Schwarzschild black hole metric as $v$ decreases and might be heuristically described as a black hole with sphaleron hair. The other solutions do not have a weak gravity limit (a limit arbitrarily close to Schwarzschild). 